\title{
3D acoustic simulation inside closed rooms bounded by non-parallel floor and ceiling surfaces
}

\author{
A. Tadeu ${ }^{1}$, I. Simões ${ }^{1}$, J. António ${ }^{1} \&$ L. Sousa ${ }^{2}$ \\ ${ }^{1}$ CICC, Department of Civil Engineering, Faculty of Sciences and \\ Technology, University of Coimbra, Portugal \\ ${ }^{2}$ ITeCons, Coimbra, Portugal
}

\begin{abstract}
A model for the simulation of the three-dimensional (3D) sound pressure level generated by acoustic sources inside closed rooms bounded by non-parallel floor and ceiling surfaces is presented. The 3D problem is solved as a sequence of two-dimensional (2D) problems with different spatial wavenumbers $k_{z}$, after a spatial Fourier transform has been applied along the $z$ direction, which is the width of the room. The problem uses a BEM formulation in the frequency domain. The use of analytically appropriate Green's functions for wedges, defined by means of an image model technique, allows the required BEM discretization to be limited to the back and front walls of the room and avoids the discretization of the other flat surfaces.

The model allows frequency dependent absorption coefficients to be defined for the various walls. In the case of the ceiling, floor and lateral room surfaces the absorption is introduced through the direct manipulation of the analytical Green's functions. The absorption of the back and front walls is simulated using impedance boundary conditions.

Keywords: boundary element method, 3D source, sound absorption, pressure level inside auditoriums.
\end{abstract}




\section{Introduction}

Analytical solutions to simulate acoustic wave propagation are only known for very simple geometries. Two basically different numerical approaches have been used to handle this problem, these being geometrical and wave-based methods.

The most popular models are based on geometrical acoustics that describe the sound propagation along sound rays, following the principles of ray optics and computer graphics. The ray tracing method $[1,2]$, the image source method $[3,4]$, beam tracing [5] and acoustic radiosity [6, 7] are some of these methods. One review of them can be found in [8]. Some of these models are also analyzed in the work of Rindel [9] on the use of computer modeling in room acoustics.

Wave based methods have been proposed and implemented using the finite element method (FEM), the finite difference method (FDM) and the boundary element method (BEM), to simulate wave related phenomena. However, they are more suitable for handling lower frequencies of excitation due to the need for the discretization of the domains. The full discretization of the domain required by the FEM and FDM leads to the definition of very fine meshes which results in a huge number of equations to solve problems at high frequencies.

The boundary element method (BEM) has long been considered a very promising technique in acoustics, since it allows the simulation of fields in unbounded domains and automatically satisfies the radiation condition at infinity [10]. Many references can be found related to its use in the time and frequency domains to solve acoustic problems (see, for example, $[11,13]$ ). The BEM, is a more efficient method in terms of computer cost as it only requires the discretization of the boundaries [11]. However as the number of elements increases with the frequency, due to the imposition at each frequency of a relation between the wavelength and the length of the boundary elements, the calculation at high frequencies can be unaffordable.

In the present paper the (3D) sound pressure level generated by acoustic sources inside closed rooms, bounded by non-parallel floor and ceiling surfaces, is modelled using the BEM in the frequency domain. The $3 \mathrm{D}$ problem is solved as a sequence of two-dimensional (2D) problems with different spatial wavenumbers $k_{z}$, after a spatial Fourier transform has been applied along the $z$ direction, which is the width of the room. When applying an inverse spatial Fourier transform to the $2 \mathrm{D}$ results to synthesize the $3 \mathrm{D}$ wavefield, the lateral walls are taken into account by using virtual sources placed so as to ensure the required boundary conditions.

The use of appropriate Green's functions for wedges, defined by means of an image model technique $[12,14]$, allows the required BEM discretization to be limited to the back and front walls of the room and to a vertical surface coupling two subdomains, avoiding the discretization of the other flat surfaces. A compact mathematical description of these Green's functions is given in this work. All image sources are placed over a circle and only the visible sources are taken into account.

This paper is organized as follows. The problem is defined in section 2, where the boundary element method formulation, the proposed Green's functions and 
the method used to simulate the sound absorption are presented. Numerical examples demonstrating the applicability and efficiency of the BEM algorithm for the simulation of a three-dimensional pressure wavefield inside a threedimensional auditorium are presented in Section 3.

\section{Problem definition}

Consider a closed room bounded by non-parallel floor and ceiling surfaces (see fig. 1a)). An harmonic point pressure source, at position $\left(x_{0}, y_{0}, z_{0}\right)$, oscillating with a frequency $\omega$, illuminates this space, creating the propagation of threedimensional acoustic waves, that travel with constant velocity $c$. The back and front walls, the ceiling and the floor are assumed to be parallel to the $z$ axis. The lateral walls are perpendicular to the $z$ axis. All surfaces may exhibit sound absorbent properties.

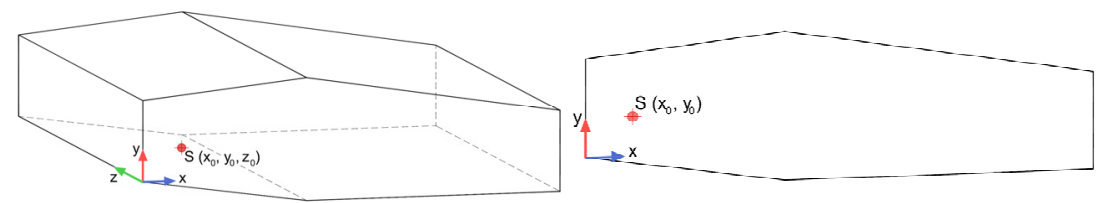

a)

b)

Figure 1: Definition of the problem: a) 3D geometry; b) 2D geometry.

The resulting incident pressure field at the receiver placed at $(x, y, z)$ is given by

$$
\hat{p}_{i n c}=\frac{\mathrm{e}^{\mathrm{i} \frac{\omega}{c}\left(c t-r_{1}\right)}}{r_{1}}
$$

in which the subscript inc denotes the incident field, $r_{1}$ is the distance from the source and $i=\sqrt{-1}$. In the absence of the lateral walls, the geometry of the problem is constant along one direction $(z)$, the incident field can be computed as a discrete summation of two-dimensional pressure fields given by

$$
p_{\text {inc }}\left(\omega, x, y, k_{z}\right)=\frac{-\mathrm{i}}{2} \mathrm{H}_{0}\left(k_{c} r_{01}\right)
$$

where $r_{01}=\sqrt{\left(x-x_{0}\right)^{2}+\left(y-y_{0}\right)^{2}}, \quad k_{c}=\sqrt{\frac{\omega^{2}}{c^{2}}-k_{z}^{2}}$ with $\operatorname{Im} k_{c}<0, k_{z}=\frac{2 \pi}{L} m$ $(m=0,-1,1,-2,2, \ldots .-M, M)$ being the spatial wavenumber, obtained after Fourier-transforming eqn (1) in the $z$ direction, $L$ is the distance between virtual sources placed along the $z$ direction in order to obtain the solution as a discrete summation and $H_{n}(\ldots)$ are second Hankel functions of order $n$. The 
problem is periodic in the $z$ dimension, and the term $\mathrm{e}^{-\mathrm{i} k_{z} z}$ is implicit in eqn (2).

Similarly, the scattered field originated by a point pressure load can be evaluated by solving a sequence of two-dimensional problems, as illustrated in Fig. 1b). The lateral walls can be taken into account later by using image sources placed so as to ensure the boundary conditions required (null pressure flows).

\subsection{Boundary element method formulation}

We first describe how the BEM is formulated to obtain the scattered acoustic pressure wave field, i.e. the pressure in the $2 \mathrm{D}$ medium generated by a spatially sinusoidal harmonic line load. The acoustic medium is first assumed to be confined by rigid boundaries. The simulation of the sound absorption is presented afterwards.

The use of Green's functions that satisfy the boundary conditions of the wedge, allows the discretization to be limited to the vertical interfaces between wedges, i.e. the back and front walls (see fig. 2), which required a substantially lower number of boundary elements.

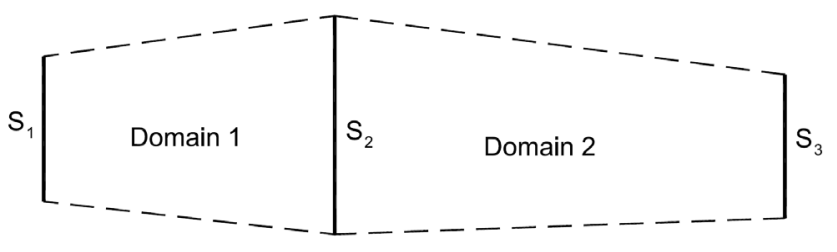

Figure 2: $\quad$ Geometry of the BEM model.

The classical boundary integral equation can be derived from the Helmholtz equation in the frequency domain by applying the reciprocity theorem, leading to:

along the interior domain 1:

$$
\begin{aligned}
b p\left(x_{0}, y_{0}, k_{z}, \omega\right)= & \int_{S_{2}} q\left(x, y, \mathbf{n}_{n 1}, k_{z}, \omega\right) G\left(x, y, x_{0}, y_{0}, k_{z}, \omega\right) \mathrm{d} s_{2} \\
& -\int_{S_{1}} H\left(x, y, \mathbf{n}_{n 1}, x_{0}, y_{0}, k_{z}, \omega\right) p\left(x, y, k_{z}, \omega\right) \mathrm{d} s_{1} \\
& -\int_{S_{2}} H\left(x, y, \mathbf{n}_{n 1}, x_{0}, y_{0}, k_{z}, \omega\right) p\left(x, y, k_{z}, \omega\right) \mathrm{d} s_{2} \\
& +p_{\text {inc }}\left(x_{0}, y_{0}, x_{s}, y_{s}, k_{z}, \omega\right),
\end{aligned}
$$

assuming that the source is within this domain. 
along the interior domain 2:

$$
\begin{aligned}
b p\left(x_{0}, y_{0}, k_{z}, \omega\right)= & \int_{S_{2}} q\left(x, y,-\mathbf{n}_{n 1}, k_{z}, \omega\right) G\left(x, y, x_{0}, y_{0}, k_{z}, \omega\right) \mathrm{d} s_{2} \\
& -\int_{S_{2}} H\left(x, y,-\mathbf{n}_{n 1}, x_{0}, y_{0}, k_{z}, \omega\right) p\left(x, y, k_{z}, \omega\right) \mathrm{d} s_{2} \\
& -\int_{S_{3}} H\left(x, y, \mathbf{n}_{n 1}, x_{0}, y_{0}, k_{z}, \omega\right) p\left(x, y, k_{z}, \omega\right) \mathrm{d} s_{3},
\end{aligned}
$$

where $G$ and $H$ represent the Green's functions for the pressure $(p)$ and pressure gradient $(q)$ at a point $(x, y)$ on the boundary $S\left(S=S_{1}+S_{2}+S_{3}\right)$ due to a virtual point pressure source at a collocation point $\left(x_{0}, y_{0}\right) . p_{\text {inc }}$ is the incident pressure field at $\left(x_{0}, y_{0}\right)$, generated by a source located in domain 1 at $\left(x_{s}, y_{s}\right) . \mathbf{n}_{n_{1}}$ represents the unit outward normal along the boundary $S$, at $(x, y)$, defined by the vector $\mathbf{n}_{n 1}=\left(\cos \theta_{n 1}, \sin \theta_{n 1}\right)$. The factor $b$ takes the value $1 / 2$ if $\left(x_{0}, y_{0}\right) \in S$ and $S$ is smooth.

The final system of equations is assembled assuring the continuity of pressures and pressure gradients along $S_{2}$. The global solution is found by solving eqns (3)-(4). This requires the discretization of the interface $S=S_{1}+S_{2}+S_{3}$.

In this analysis the interface is discretized with straight boundary elements, with one nodal point in the middle of each element. The unknown nodal pressures and pressure gradients are obtained by solving this system of equations, allowing the pressure field along the domain to be defined.

The required integrations in eqns (3)-(4) are evaluated using a Gaussian quadrature scheme when they are not performed along the loaded element. For the loaded element, the existing singular integrands of the Green's functions are calculated analytically, following the expressions in Tadeu et al. [15].

\subsection{Sound absorption simulation}

The sound absorption in the back and front walls can be simulated by prescribing boundary conditions that relate the pressure with the velocity at each collocation point. This can be viewed as a Robin boundary condition (impedance boundary condition), that is

$$
q\left(x, y, \mathbf{n}_{n 1}, k_{z}, \omega\right)=-\mathrm{i} \omega \rho \frac{1}{\mathrm{Z}(\omega)} p\left(x, y, k_{z}, \omega\right) .
$$

\subsection{Green's functions for wedges}

To avoid the discretization of the non-parallel rigid floor and ceiling the Green's functions and the incident pressure need to be expressed in a way that satisfy 
null normal velocities at those boundaries. This can be accomplished by adding the pressure field generated by the real source to that produced by virtual sources (image sources), which are located in such a way that they act as mirrors of the real source in relation to those planes.

Consider a wedge with an opening angle (see fig. 3).

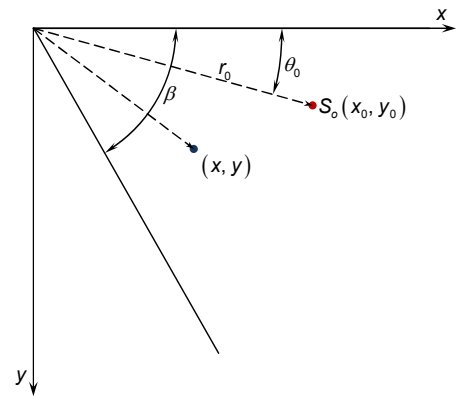

Figure 3: Wedge geometry.

For simplicity the origin of the Cartesian coordinate system is placed at its corner. When the source is placed at point $\left(x_{0}, y_{0}\right)$ the response at the receiver placed at $(x, y)$ is given by

$$
G\left(x, y, x_{0}, y_{0}, k_{z}, \omega\right)=\frac{-\mathrm{i}}{4} \mathrm{H}_{0}\left(k_{c} r_{01}\right)-\frac{\mathrm{i}}{4} \sum_{n=1}^{N S}\left[\sum_{j=1}^{4} H_{0}\left(k_{c} r_{j}\right)\right]
$$

with $\quad r_{j}=\sqrt{\left[x-r_{0} \cos \left(\gamma_{j}\right)\right]^{2}+\left[y-r_{0} \sin \left(\gamma_{j}\right)\right]^{2}}, \quad$ where $\quad \gamma_{1}=-\theta_{0}-2 \beta(n-1)$, $\gamma_{2}=-\theta_{0}+2 \beta n, \gamma_{3}=\theta_{0}-2 \beta n, \gamma_{4}=\theta_{0}+2 \beta n$ and $\theta_{0}$ is the azimuth distance of the source.

$N S$ is associated with the number of sources generating the responses that reach the receivers within the time interval fixed by the frequency increment in the calculation. The number of image sources considered is finite because only the observable images or audible image sources are taken into account [8]. Those surfaces can also be simulated as absorbent ones. This is accomplished by multiplying the reflected pressure generated by the mirrored image source at those surfaces by a reflection coefficient $\sqrt{1-\alpha}$, calculated from the sound absorption coefficient $\alpha$.

\subsection{Simulation of the lateral walls}

The lateral walls, perpendicular to the $z$ axis, are simulated using the image source technique. In their absence, the geometry of the stated problem does not vary along the longitudinal $z$ direction. Thus, the 3D incident field can be formulated as a summation of $2 \mathrm{D}$ solutions after applying a Fourier 
transformation along that direction. Therefore, the solutions are first obtained for the $2 \mathrm{D}$ cross-section for varying axial wavenumbers, as explained above. Then the lateral walls are simulated by applying an image source technique and the $3 \mathrm{D}$ solution is calculated by applying an inverse spatial Fourier Transform.

\section{Case study}

The pressure wave propagation in a three-dimensional auditorium is simulated by means of the boundary element formulation proposed and verified above. The geometry is shown in fig. 4. Its longitudinal 2D cross-section is the same as above. The room has a width of $15.0 \mathrm{~m}$. This host medium is excited by a 3D harmonic point load located at point $\mathrm{S}(5.0 \mathrm{~m}, 1.8 \mathrm{~m}, 3.0 \mathrm{~m})$.

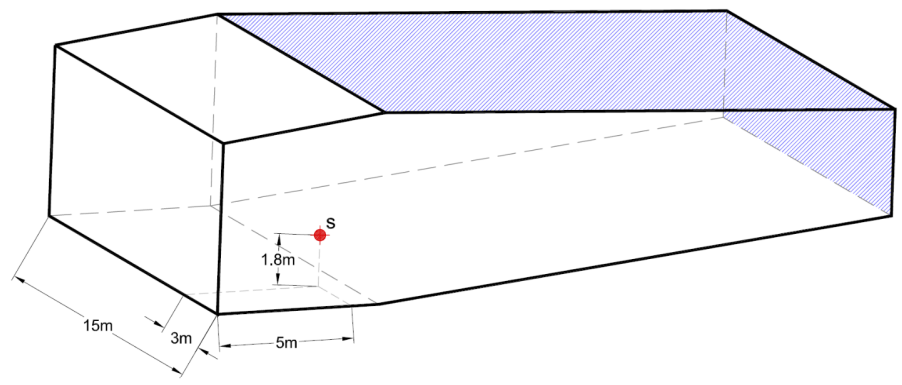

Figure 4: Geometry of the auditorium.

As above, only the vertical interfaces between wedges, the back and front walls, need to be discretized to obtain the two-dimensional solution. As above, the number of boundary elements is defined by the relation between the wavelength $(\lambda)$ and the length of the boundary elements $(L)$, which is set to 8 . The minimum number of boundary elements used for discretizing each surface was 30, at an initial frequency.

Frequency results were computed in the range $8.0 \mathrm{~Hz}$ to $1024.0 \mathrm{~Hz}$, with time responses obtained by applying an inverse (fast) Fourier transform in $\omega$, using a Ricker pulse with a characteristic frequency of $350.0 \mathrm{~Hz}$ for the source time evolution. A frequency increment of $8.0 \mathrm{~Hz}$ specifies a total time window of $0.125 \mathrm{~s}$.

Time results in terms of pressures were determined in fine grids of receivers placed over five planes corresponding to: $x=5.925 \mathrm{~m}, \quad x=13.425 \mathrm{~m}$, $x=20.925 \mathrm{~m}, y=2.925 \mathrm{~m}$ and $z=8.0 \mathrm{~m}$ (fig. 5). The receivers were spaced at equal intervals of $0.15 \mathrm{~m}$ along the $x, y$ and $z$ directions.

The effect of the absorption in the acoustic wavefield is illustrated by assuming that the back wall and ceiling (see fig. 4) of the room have a constant sound absorption coefficient at all frequencies equal to $\alpha=0.8$ (although the 
model allows the absorption coefficients to vary with the frequency) while the remaining flat boundaries are rigid. The results are compared with those obtained when all the boundaries are perfectly reflective.

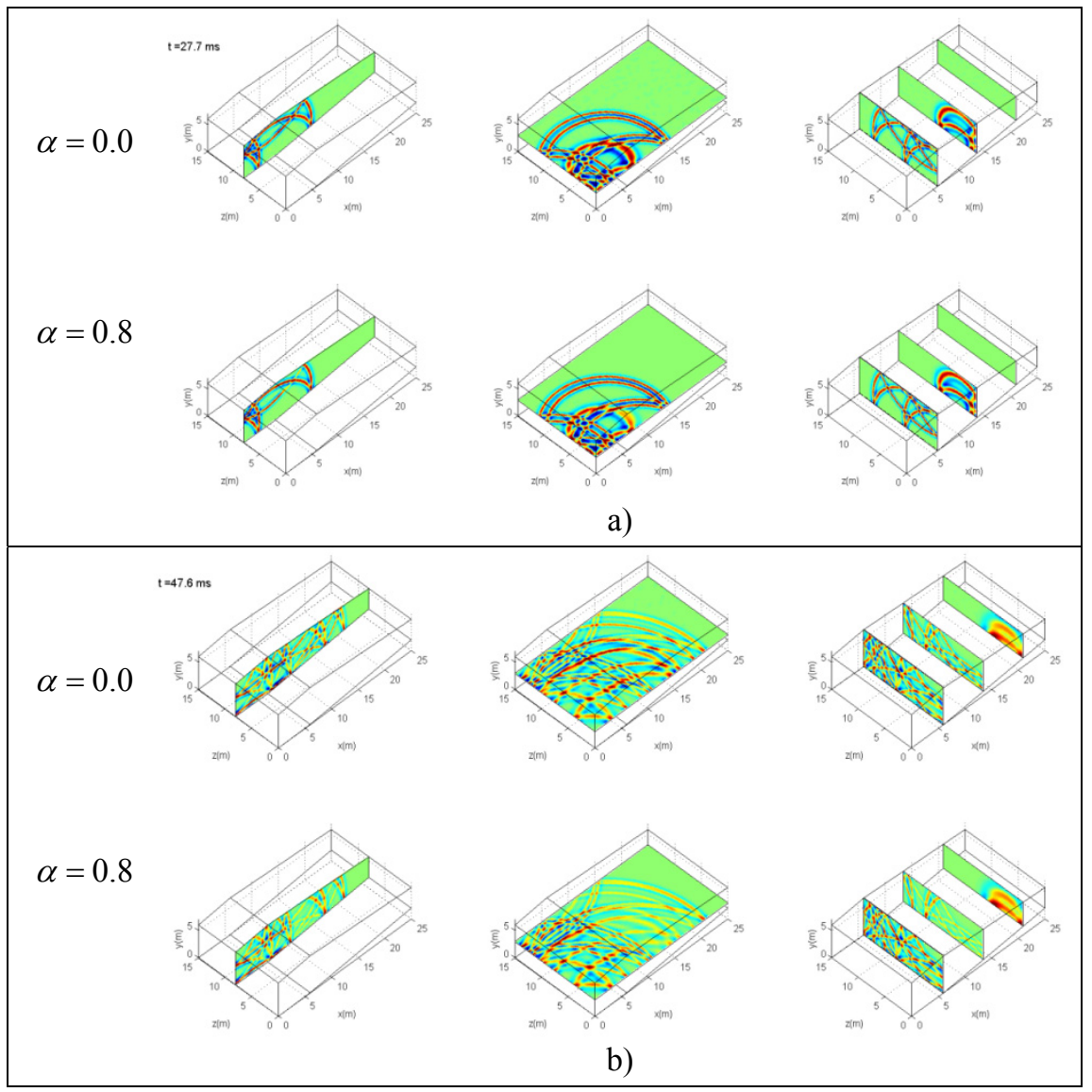

Figure 5: Snapshots of responses as at $t=27.7 \mathrm{~ms}$ (a) and $t=47.6 \mathrm{~ms}$ (b).

The results are illustrated by a set of 3D snapshots captured at different time instants from computer animations. In these 3D snapshots of pressure, a color scale is used in which the red and blue shades correspond respectively to higher and lower values of pressure amplitude.

\section{Conclusions}

A model for simulating the 3D acoustic sound wave propagation inside rooms with non-parallel floor and ceiling surfaces has been presented. A BEM formulation was used to solve the problem for as a sequence of two-dimensional 
(2D) problems for different spatial wavenumbers across the width of the room. Appropriate Green's functions for wedges, defined by means of an image model technique, were incorporated in this formulation in order to reduce the number of boundary elements needed to discretize the geometry of the problem. Thus the computational cost involved to model the problem is substantially lower. The model allows different frequency-dependent sound absorption coefficients to be ascribed to the boundary surfaces of the room.

\section{References}

[1] Kulowski, A., Error investigation for the ray tracing technique. Applied Acoustics, 15(4), pp. 263-274, 1982.

[2] Kulowski, A., Algorithmic representation of the ray tracing Technique. Applied Acoustics, 18(6), pp. 449-469, 1985.

[3] Allen, J.B. and Berkley, D.A., Image method for efficiently simulating small room acoustics. Journal of Acoustical Society of America, 65(4), pp. 943-950, 1979.

[4] Lee, H. and Lee, B.H., An efficient algorithm for the image model technique. Applied Acoustics, 24(2), pp. 87-115, 1988.

[5] Laine S., Siltanen, S., Lokki, T. and Savioja, L., Accelerated beam tracing algorithm. Applied Acoustics, 70(1), pp. 172-181, 2009.

[6] Nosal, E.M., Hodgson, M. and Ashdown, I., Improved algorithms and methods for room sound-field prediction by acoustical radiosity in arbitrary polyhedral rooms. Journal of the Acoustical Society of America, 116(2), pp. 970-980, 2004.

[7] Hodgson, M. and Nosal, E.M., Experimental evaluation of radiosity for room sound-field prediction. Journal of the Acoustical Society of America, 120(2), pp. 808-819, 2006.

[8] Vorländer, M., Auralization, Springer-Verlag: Berlin, 2007.

[9] Rindel, J.H., The use of computer modeling in room acoustics. Journal of Vibroengineering, 3(4), pp. 41-72, 2000.

[10] Ciskowski, R.D. and Brebbia, C.A., (eds). Boundary Element Methods in Acoustics, Computational Mechanics Publications and Elsevier Applied Science: Southampton, 1991.

[11] Wu, T.W., (ed). Boundary Element Acoustics - fundamentals and computer codes, Southampton: WIT Press, 2000.

[12] António, J., Tadeu, A. and Godinho, L., 2.5D scattering of waves by rigid inclusions buried under a fluid channel via BEM. European Journal of Mechanics /A Solids, 24(6), pp. 957-973, 2005.

[13] Tadeu, A. and Godinho, L., Scattering of acoustic waves by movable lightweight elastic screens. Engineering Analysis with Boundary Elements, 27(3), pp. 215-226, 2003.

[14] António, J., Tadeu, A. and Amado Mendes, P., A 2.5D traction boundary element method formulation applied to the study of wave propagation in a fluid layer hosting a thin rigid body. Journal of Computational Acoustics, 16(2), pp. 177-198, 2008. 
294 Boundary Elements and Other Mesh Reduction Methods XXXIV

[15] Tadeu, A., Santos, P.F.A. and Kausel, E., Closed-form integration of singular terms for constant, linear and quadratic boundary elements. Part I. SH wave propagation. Engineering Analysis with Boundary Elements, 23(8), pp. 671-681, 1999. 